\title{
ESTUDIO DEMOGRÁFICO DEL LIRIO ACUÁTICO EICHHORNIA CRASSIPES (MART) SOLMS: DINÁMICA DE CRECIMIENTO EN DOS LOCALIDADES SELECTAS DE MÉXICO*
}

\author{
MARTHA S. NIÑO SULKOWSKA** y ANTONIO LOT**
}

\begin{abstract}
RESUMEN
No obstante el gran auge generado en los últimos años en torno al lirio acuático, ya sea por su papel negativo o bien por su utilidad potencial, se ha omitido visualizar la problemática del crecimiento desmesurado de esta maleza dentro del contexto de la ecología poblacional, desconociéndose muchos aspectos sobre el comportamiento de las poblaciones clonales en condiciones naturales. Como una aportación hacia la comprensión de los mecanismos que regulan la estructura de las poblaciones de $E$. crassipes y a partir de una perspectiva de organización modular, se describe la dinámica de crecimiento en dos localidades selectas de México. En contraste al intenso crecimiento de la población exhibido en condiciones de eutroficación, la producción de unidades de crecimiento clonal (ramets) está fuertemente limitada en un medio biológicamente diverso, manifestándose la gran plasticidad fenotípica de la especie en su conducta vegetativa y reproductiva.
\end{abstract}

\begin{abstract}
The amount of information concerning the water hyacint grew in a explosive way during recent years due to its potential damaging or benefic effect on the environment, nevertheless the study of the population ecology of this plant has been neglected till now. In particular, the behavior of clonal populations in natural conditions.

As a contribution to the understanding of the factors that regulate the structure of populations of Eichhornia crassipes in relation with its modular organization, we describe here the growth dynamics of the plants in two selected water bodies of Mexico.

*Recibido para publicación en julio de 1983.

**Departamento de Botánica, Instituto de Biología, Universidad Nacional Autónoma de México. Ap. Postal 70-233, 04510 México, D.F.
\end{abstract}

Niño-Sulkowska MS, Lot A. 1983. Estudio demográfico del lirio acuático Eichhornia crassipes (Mart) Solms: dinámica de crecimiento en dos localidades selectas de México. Boletín de la Sociedad Botánica de México 45: 71-83. 
In contrast with the fast growth shown by populations living in euthrophicated environments, the production of growth units (ramets) by the plants is strongly limitated by an ecologicaly diverse environment but the phenotipic plasticity increases mainly in the vegetative and reproductive behavior.

\section{INTRODUCCIÓN}

Uno de los temas que más ha atraído la atención en los últimos años es la controversia en torno a los beneficios o daños provocados por el lirio acuático, Eichhornia crassipes (Mart) Solms. (Vietmeyer, 1975; Bates and Hentges, 1976).

$E$. crassipes se considera como una de las malezas acuáticas mundialmente más dañinas, debido a los alarmantes efectos adversos causados en el medioambiente dulceacuícola, asociados a su extraordinario potencial de desarrollo y facilidad de dispersión (Penfound y Earle, 1948; Gay, 1958, 1960; Bishai, 1960; Jain, 1975; Soerjani et al., 1975; Guillarmod, 1979; Bayo et al., 1981; Formo y Wright, 1981). La presencia de extensos tapetes móviles, no sólo modifica la calidad y cantidad de agua, sino que su impacto trasciende a todos los niveles, desde los ecológicos y de salud pública, hasta los sociales, económicos y aún políticos. Con frecuencia se mencionan los efectos negativos inmediatos como la obstrucción del flujo de agua en los canales de irrigación, la dificultad de navegación, la destrucción y deterioramiento de puentes y presas (debido a la fuerte presión que ejerce la acumulación de plantas), notables alteraciones en la tasa de evotranspiración, la reducción en la penetración de la luz, el incremento de la turbiedad y acidez del agua, así como la modificación del balance de los principales gases disueltos (Timmer y Weldon, 1967; Almazán y Boyd, 1978; Cooley y Martín, 1980; McDonald y Wolverton, 1980). La alteración de las condiciones fisicoquímicas normales se traduce finalmente en una pérdida de la biota natural, se provee de hábitats para el desarrollo de patógenos y vectores de enfermedades como la malaria, así como se propicia la formación de las llamadas "islas flotantes", que en última instancia conducen a acelerar la sucesión de los sistemas dulceacuícolas (Hayashi et al., 1978; Trivedy et al, 1978; Rai y Jayashree, 1979; Sanders et al., 1982).

Los intensos esfuerzos dirigidos hacia la erradicación del lirio acuático, patentes en las amplias referencias y revistas especializadas sobre el tema (Hyacinth Control Journal, Advances in Pest Control Research, Weeds, Weed Science), han aportado soluciones parcialmente satisfactorias. Pocas veces los métodos de control tienen efectos específicos sobre las malezas, sin tener repercusión en el resto del sistema, otras veces son efectivos a corto plazo a expensas de un alto costo, o bien, logran mantener niveles tolerables por medio del control biológico (Hitchcock et al., 1950; Hall, 1961; Little, 1968; Perkins, 1974; Room et al., 1979; Wright, 1980; Center, 1981).

La elevada tasa de utilización de elementos esenciales, aunado a su intenso potencial de crecimiento y forma de vida, motivó una reorientación al problema del lirio, a favor de su utilización. Varios autores han señalado su eficacia en el tratamiento de aguas residuales domésticas, contribuyendo notablemente a abatir la eutroficación (Sinha y Sinha, 1969; Boyd, 1970, 1973; Roger y Davis, 1972; Dunigan et al., 1975; Ornes y Sutton, 1975; Wooten y Dodd, 1976; Cooley et al., 1978). Además de actuar como un filtro natural, los densos tapetes móviles representan a la vez tanto un recurso nutricional muy valioso como fuente de proteínas para la alimentación animal, como una materia prima para la fabricación de papel, o bien como combustible (Boyd, 1968, 1969, 


\section{DEMOGRAFÍA Y CRECIMIENTO DEL LIRIO ACUÁTICO}

1976; Little, 1968a; Bagnal et al., 1973; Wolverton y McDonald, 1978). Su aprovechamiento como mejorador de suelos (abono verde) es ampliamente conocido en el sistema de producción agrícola de las Chinampas (Lot et al., 1979; Quiroz et al., 1982). Sin embargo, el lirio también es capaz de fijar en sus tejidos pesticidas, fenoles y sobre todo metales pesados presentes en las aguas residuales, tales como el plomo, cobre, arsénico, cadmio y mercurio. La concentración de éstos elementos en el tejido vegetal sobrepasa los niveles considerados como tóxicos por las normas mundiales, lo cual restringe severamente su uso potencial (Wolverton y McKown, 1976; Stawinski y Monroy, 1982).

Es importante enfatizar que la presencia de lirio acuático como maleza, es el reflejo de un estado de perturbación provocado por el enriquecimiento de nutrientes, situación que se torna más compleja en regiones como México, en donde no existen predadores naturales que controlen su desarrollo. Por otra parte, su valor como recurso natural no se puede compensar con el impacto ecológico al ecosistema dulceacuícola, que se traduce finalmente en la pérdida de un recurso aún más esencial, el agua (Gopal y Sharma, 1979).

Bajo esta tendencia pragmática, se ha omitido el planteamiento del control del lirio acuático partiendo de su estudio dentro de un contexto demográfico, como poblaciones clonales que muestran patrones de respuesta altamente plásticos a los cambios en las condiciones medioambientales, alterando sus tasas de crecimiento, propagación vegetativa y reproducción. Un concepto fundamental para el entendimiento de la demografía de especies que se reproducen principalmente por medios vegetativos, es la idea introducida por Harper (1967), en el sentido de considerar a una planta individual como un organismo modular, constituido por una serie de unidades estructurales básicas que se repiten durante el crecimiento, a partir de un meristemo. En este sentido, el lirio acuático tiene una estructura claramente modular; cada planta individual, producto de la reproducción sexual y consecuentemente con un genotipo particular, forma a través del desarrollo estolones que se diferencían en rizomas con sus hojas, meristemos axilares, raíces en las primeras etapas de su crecimiento y eventualmente inflorescencias, formándose vástagos genéticamente similares a la planta progenitora, capaces de llevar una vida independiente. Estas unidades de crecimiento clonal, denominadas ramets, presentan ciclos de vida definidos, y al igual que en los individuos de una población (genets), se reconocen atributos tales como una estructura de edades, tiempos de vida, tasas de natalidad y mortalidad, de manera que pueda analizarse en términos demográficos esta dinámica modular o subpoblacional dada por el crecimiento individual. (Bazzaz y Harper, 1977; Harper, 1978; Harper y Bell, 1979; White, 1979).

Watson (comunicación personal) propone el estudio de las poblaciones meristemáticas de especies como $E$. crassipes, que crecen en hábitats diferentes a los sistemas terrestres y en donde las relaciones espaciales no son estables a través del tiempo. Sus trabajos de investigación bajo condiciones controladas de laboratorio son básicos como punto de partida para el entendimiento del comportamiento de las poblaciones en su medio natural.

La inquietud de plantear nuevas alternativas de control para el lirio acuático, motivaron examinar la estructura y mecanismos de regulación de las poblaciones de $E$. crassipes en algunas localidades selectas de México. Como una aportación en este sentido, se describen las características de crecimiento exhibidas por diferentes clones y las modificaciones en su conducta vegetativa y reproductiva, cuando se exponen a condiciones medioambientales contrastantes. 


\section{MATERIAL Y MÉTODOS}

Se seleccionaron dos sitios que representan condiciones en donde el lirio se desarrolla en forma contrastante (tabla 1). El primer sitio permanente de estudio, sitio $\mathrm{Hi}$ dalgo, se localiza en una zona templada en donde se reservó un depósito de agua proveniente de la Presa Requena, Hidalgo. Presenta una superficie de $200 \mathrm{~m}^{2}$ y una profundidad variable entre 1 y $3 \mathrm{~m}$, lo que condiciona marcadas fluctuaciones diarias de temperatura y una acción directa del viento. Como segundo sitio permanente de estudio, sitio Veracruz, se eligió un estanque natural formado entre dunas, característico de la zona cálida adyacente a la costa.

TABLA 1. Localización, características altitudinales y climáticas de los sitios de estudio y localidades de origen del material

\begin{tabular}{|c|c|c|c|c|}
\hline \multirow[b]{2}{*}{ Localidad } & \multicolumn{2}{|c|}{ Ubicación } & \multirow{2}{*}{$\begin{array}{l}\text { Altitud } \\
(m)\end{array}$} & \multirow[b]{2}{*}{ Clima* } \\
\hline & Lat. $N$ & Long. $W$ & & \\
\hline Sitio Hidalgo & $19^{\circ} 54^{\prime}$ & $99^{\circ} 19^{\prime}$ & 2190 & Templado semiseco $\mathrm{BS}_{1} \mathrm{k}^{\prime} \mathrm{w}(\mathrm{w})\left(\mathrm{i}^{\prime}\right) \mathrm{g}$ \\
\hline Presa Requena & $19^{\circ} 54^{\prime}$ & $99^{\circ} 19^{\prime}$ & 2109 & Templado semiseco $\mathrm{BS}_{1} \mathrm{k}^{\prime} \mathrm{w}(\mathrm{w})\left(\mathrm{i}^{\prime}\right) \mathrm{g}$ \\
\hline Sitio Veracruz & $19^{\circ} 11^{\prime}$ & $96^{\circ} 15^{\prime}$ & 50 & Cálido subhúmedo $\mathrm{Aw}{ }_{1}(\mathrm{w})(\mathrm{i})$ \\
\hline Lag. Alvarado & $18^{\circ} 53^{\prime}$ & $95^{\circ} 56^{\prime}$ & 0 & Cálido subhúmedo $\mathrm{Aw}^{\prime \prime}{ }_{2}(\mathrm{w})(\mathrm{i})^{\prime} \mathrm{g}$ \\
\hline
\end{tabular}

*Sistema de clasificación climática de Köeppen, modificado por García (1973).

La elección de poblaciones geográfica, climática y altitudinalmente diferentes, y que en su medio natural exhiben diferentes patrones de desarrollo, asegura en cierto grado trabajar con genotipos diferentes, a nivel interclonal. La procedencia de los clones son la Presa Requena (clon Hidalgo) y la Laguna de Alvarado (clon Veracruz).

Inicialmente las plantas se dejaron desarrollar en su medio original con objeto de contar con un número suficiente de ramets o vástagos de origen conocido y así seleccionar los más adecuados para el trasplante por su tamaño y edad relativa. El número de ramets originales se dedujo a partir de la relación de la varianza del número de ramets producidos por diferentes plantas, después de 195 días de observación (Fig. 1).

A partir de transplantes recíprocos en cada sitio se contaba con dos clones representados originalmente por 13 ramets cada uno, los cuales se dejaron desarrollar en condiciones naturales, mezclados entre sí y en baja densidad (menos de 10 plantas $\mathrm{m}^{2}$ ), a lo largo de 8 meses (de mayo a diciembre, 1982).

Para el marcaje y registro de parámetros se requirió estar en contacto directo con las plantas, tratando de provocar la menor alteración posible. Cada 15 días y posteriormente cada mes se marcaban las nuevas generaciones de ramets y se registraban diferentes índices de crecimiento y reproducción como la altura de las plantas, el número de hojas funcionales, la producción de hojas, vástagos e inflorescencias, así como apreciaciones de la coloración y algún tipo de daño en las plantas.

El procesamiento de los datos se basó en pruebas de ajuste para hallar la función estadística más significativa que describa el crecimiento de las poblaciones (regresión lineal, exponencial y logística). 


\section{DEMOGRAFÍA Y CRECIMIENTO DEL LIRIO ACUÁTICO}

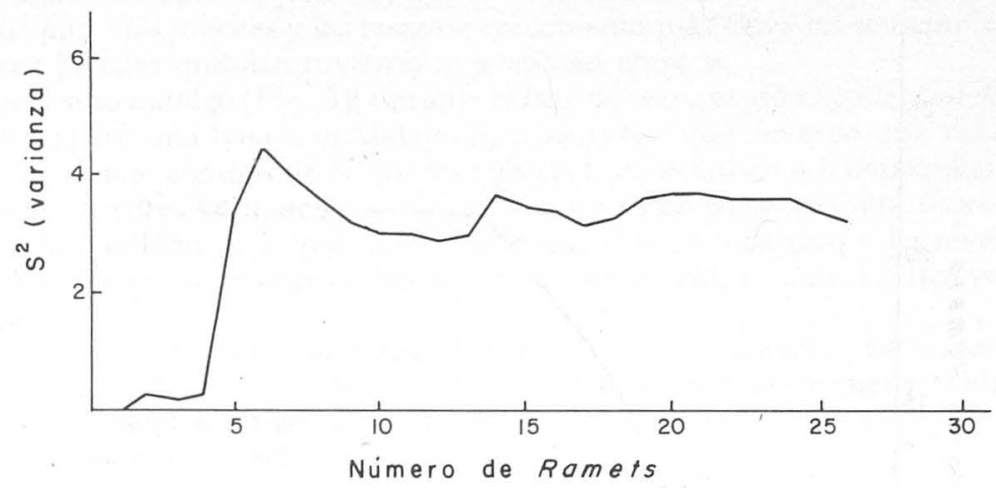

Fig. 1. Significancia del tamaño de muestra.

\section{RESULTADOS}

Producción y supervivencia de unidades de crecimiento clonal (ramets)

El crecimiento de los clones bajo las diferentes condiciones de desarrollo se ajusta de manera muy significativa (tablas 2, 3 y 4), al comportamiento de la función logística:

$$
\mathrm{N}_{\mathrm{t}}=\frac{\mathrm{K}}{1+\mathrm{e}^{-\mathrm{rt}\left(\mathrm{K} / \mathrm{No}_{0}-1\right)}},
$$

en donde $\mathrm{N}$, representa el número de ramets producidos; No, el número inicial de ramets; t, el tiempo de observación; r, la tasa de incremento natural y $\mathrm{K}$, el valor límite de crecimiento de la población o capacidad de carga del medio ambiente (Harper, 1977).

En las condiciones del sitio Hidalgo (Fig. 2), el límite de crecimiento se alcanza a densidades notablemente altas, sobre todo para el clon Hidalgo que presenta una fase de incremento más prolongada, formándose hasta 5 generaciones sucesivas de ramets. En contraste, en el sitio Veracruz el crecimiento de los clones está limitado a densidades relativamente bajas, sin manifestarse diferencias considerables entre los clones.

La tasa intrínseca de crecimiento es ligeramente mayor para el clon Veracruz, en ambos sitios de estudio, en relación a los valores exhibidos por el clon Hidalgo.

Considerando el número inicial de ramets por clon (tabla $2, \mathrm{~T}=13$ ramets), el tamaño de la muestra no es suficiente para establecer diferencias intraclonales estadísticamente significativas, sin embargo, cuando los clones se desarrollan en un medioambiente diferente del original, los ramets que llevan una vida independiente al perderse la unión con la planta progenitora $(\mathrm{S})$ exhiben tasas de crecimiento relativamente más elevadas que los que mantuvieron una unidad clonal (U). Por otra parte, los valores más altos para el límite de crecimiento se registraron entre los ramets del clon Hidalgo que mantuvieron una unidad entre sí, al desarrollarse tanto en las condiciones del sitio Hidalgo como en las del sitio Veracruz. 


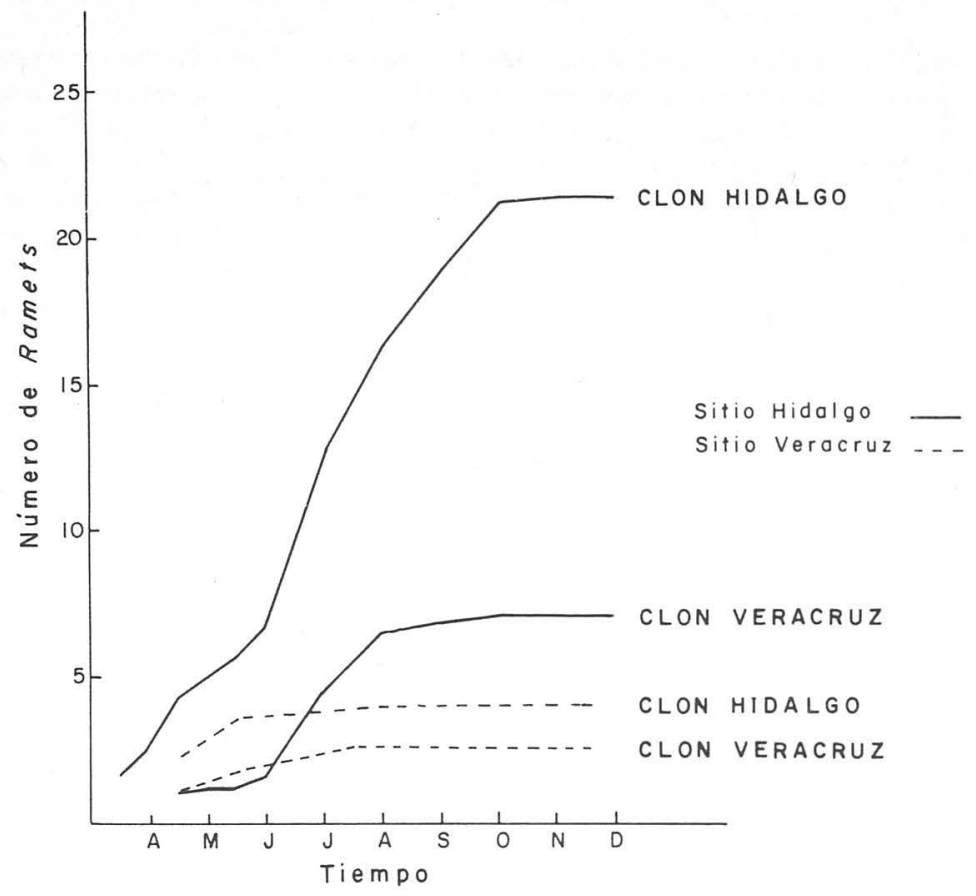

Fig. 2. Producción de unidades de crecimiento clonal.

TABLA 2. Parámetros, ajuste y significancia $\left(-\mathrm{P}>0.05 ;{ }^{*} \mathrm{P}<0.05 ; * * \mathrm{P}<0.01\right)$ de la función logística que describe el crecimiento de clones de E. crassipes

Sitio

\begin{tabular}{|c|c|c|c|c|c|c|c|}
\hline & & $K$ & $r$ & No & $R^{2}$ & $F$ & \\
\hline \multirow{3}{*}{$\begin{array}{l}\text { Clon } \\
\text { Hidalgo }\end{array}$} & $\mathrm{T}$ & 23.6 & 0.016 & 3.8 & 0.94 & 64.7 & ** \\
\hline & $\mathrm{S}$ & 19.7 & 0.024 & 2.1 & 0.98 & 184.8 & $* *$ \\
\hline & U & 26.5 & 0.029 & 1.3 & 0.96 & 115.9 & ** \\
\hline
\end{tabular}

Hidalgo

Sitio

$\begin{array}{lcrrrrrr} & \mathrm{T} & 7.7 & 0.030 & 0.5 & 0.96 & 94.4 & * * \\ \text { Clon } & \mathrm{S} & 8.2 & 0.029 & 0.4 & 0.98 & 159.1 & \text { ** } \\ \text { Veracruz } & \mathrm{U} & 7.6 & 0.022 & 0.8 & 0.83 & 16.6 & \text { ** }\end{array}$

Veracruz

\begin{tabular}{llllllll} 
& $\mathrm{T}$ & 2.7 & 0.032 & 0.7 & 0.97 & 59.1 & $* *$ \\
Clon & $\mathrm{S}$ & 2.7 & 0.031 & 0.7 & 0.98 & 80.1 & ** \\
Veracruz & $\mathrm{U}$ & 2.8 & 0.029 & 0.5 & 0.97 & 57.9 & ** \\
\hline
\end{tabular}




\section{DEMOGRAFÍA Y CRECIMIENTO DEL LIRIO ACUÁTICO}

También se aprecia (tabla 3) que el valor límite de crecimiento disminuye en las generaciones más jóvenes y las tasas de crecimiento más elevadas se manifiestan entre los ramets jóvenes que mantuvieron una unidad entre sí.

En el sitio hidalgo (Fig. 3), durante la fase de incremento exponencial de la población se registró una baja mortalidad entre los ramets que llevaron una vida independiente, en donde algunas de las plantas jóvenes no alcanzaron a desarrollarse, siendo abortadas en corto tiempo. A partir de octubre se produce una alta mortalidad que afecta a la totalidad de la población, asociada al brusco descenso del nivel del agua y las bajas temperaturas prevalescientes. La mortalidad, es más drástica para el clon foráneo.

En el sitio Veracruz la mortalidad es frecuente desde las primeras etapas del crecimiento de la población entre los ramets originales, las plantas jóvenes son más tolerantes y de ellas depende el mantenimiento de la población.

TABLA 3. Parámetros, ajuste y significancia de la función logística que describe la producción de ramets por generación (0, I, II, III y IV: ramets originales, 1er, 2da, 3er y 4a generación)

\begin{tabular}{|c|c|c|c|c|c|c|c|c|c|}
\hline & & & & $K$ & $r$ & No & $R^{2}$ & $F$ & \\
\hline \multirow{15}{*}{$\begin{array}{l}\text { Sitio } \\
\text { Hidalgo }\end{array}$} & \multirow{9}{*}{$\begin{array}{l}\text { Clon } \\
\text { Hidalgo }\end{array}$} & \multirow{5}{*}{$\mathrm{S}$} & 0 & 5.9 & 0.028 & 1.4469 & 0.93 & 63.04 & ** \\
\hline & & & I & 1.3 & 0.041 & 0.0617 & 0.95 & 85.80 & ** \\
\hline & & & II & 0.4 & 0.027 & 0.2941 & 0.80 & 18.00 & $* *$ \\
\hline & & & III & 0.3 & 0.039 & 0.0004 & 0.95 & 85.50 & $* *$ \\
\hline & & & IV & 0.1 & 0.040 & 0.0001 & 0.89 & 36.00 & $* *$ \\
\hline & & \multirow{4}{*}{$\mathrm{U}$} & 0 & 5.3 & 0.026 & 1.1365 & 0.84 & 24.37 & $* *$ \\
\hline & & & I & 2.0 & 0.027 & 0.1416 & 0.91 & 43.55 & ** \\
\hline & & & II & 0.8 & 0.062 & 0.0064 & 0.99 & 346.90 & $* *$ \\
\hline & & & III & 0.2 & 0.075 & 0.0003 & 0.71 & 11.07 & ** \\
\hline & \multirow{6}{*}{$\begin{array}{l}\text { Clon } \\
\text { Veracruz }\end{array}$} & \multirow{3}{*}{$\mathrm{S}$} & 0 & 4.0 & 0.061 & 0.0117 & 0.97 & 132.42 & $* *$ \\
\hline & & & I & 0.6 & 0.063 & 0.0014 & 0.96 & 92.42 & $* *$ \\
\hline & & & II & 0.2 & 0.028 & 0.0002 & 0.83 & 20.74 & $* *$ \\
\hline & & \multirow{3}{*}{$\mathrm{U}$} & 0 & 3.5 & 0.029 & 0.2070 & 0.77 & 11.99 & $* *$ \\
\hline & & & I & 0.7 & 0.620 & 0.0013 & 0.95 & 169.14 & $* *$ \\
\hline & & & II & 0.1 & 0.053 & 0.0001 & 0.94 & 51.33 & ** \\
\hline \multirow{8}{*}{$\begin{array}{l}\text { Sitio } \\
\text { Verácruz }\end{array}$} & \multirow{4}{*}{$\begin{array}{l}\text { Clon } \\
\text { Hidalgo }\end{array}$} & \multirow{2}{*}{$\mathrm{S}$} & 0 & 1.9 & 0.050 & 0.9375 & 0.93 & 25.47 & ** \\
\hline & & & I & 0.3 & 0.026 & 0.2434 & 0.50 & 2.0 & - \\
\hline & & \multirow{2}{*}{$\mathrm{U}$} & 0 & 0.0 & 0.000 & 0.0000 & 0.00 & 000.00 & - \\
\hline & & & I & 0.6 & 0.066 & 0.0006 & 0.99 & 317.79 & ** \\
\hline & \multirow{4}{*}{$\begin{array}{l}\text { Clon } \\
\text { Veracruz }\end{array}$} & \multirow[b]{2}{*}{ S } & 0 & 1.2 & 0.075 & 0.0407 & 0.98 & 88.69 & $* *$ \\
\hline & & & I & 0.3 & 0.077 & 0.0002 & 0.96 & 47.40 & ** \\
\hline & & \multirow{2}{*}{$\mathrm{U}$} & 0 & 1.2 & 0.022 & 0.2343 & 0.94 & 30.07 & ** \\
\hline & & & I & 0.6 & 0.069 & 0.0013 & 0.93 & 27.99 & $* *$ \\
\hline
\end{tabular}


Producción de hojas y altura de los ramets

La producción de hojas exhibe un comportamiento muy homogéneo (tabla 4). Bajo condiciones que favorecen el crecimiento de la población, la producción de hojas por las plantas jóvenes que aún no forman vástagos es relativamente elevada mientras que el límite de producción se alcanza a densidades más bajas, en comparación al compor-

\section{CLON HIDALGO CLON VERACRUZ}

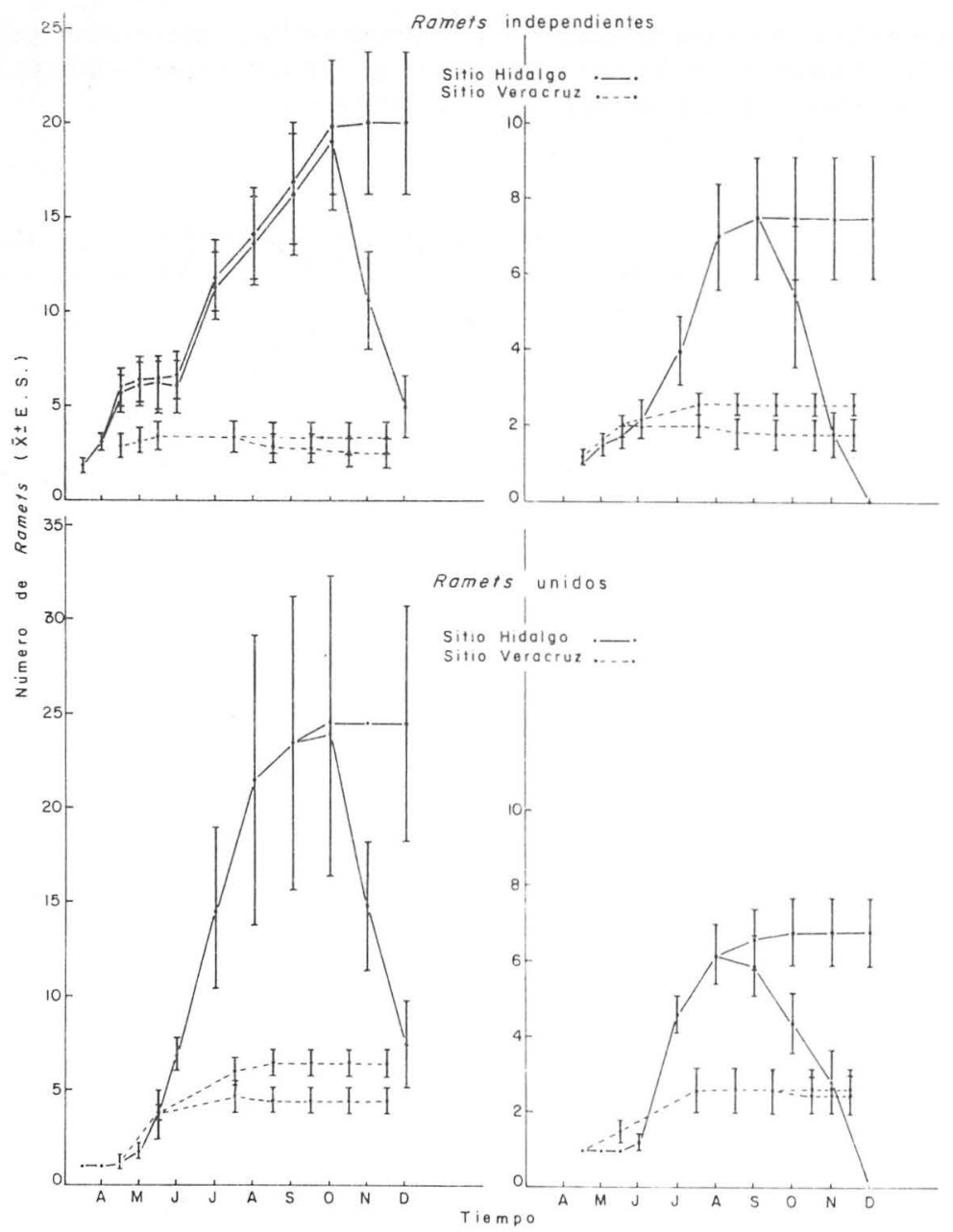

Fig. 3. Número de ramets producidos y supervivientes, bajo diferentes condiciones de desarrollo. 


\section{DEMOGRAFÍA Y CRECIMIENTO DEL LIRIO ACUÁTICO}

tamiento de las plantas más viejas en donde la tasa de producción es menor y se alcanzan los valores más altos para el límite de producción. Cuando la producción de unidades de crecimiento clonal está limtada, no se aprecian diferencias en el valor límite de producción de hojas entre las generaciones de ramets, registrándose en general altos valores.

El número de hojas funcionales por ramets varía entre 4 y 6 ; el mantenimiento de un arreglo mayor (de 6 a 8) solo se presentó cuando el clon Veracruz se desarrolla en condiciones originales (sitio Veracruz).

Una tendencia común es la uniformidad alcanzada en el tamaño de los ramets, con alturas de $5 \mathrm{~cm}$.

\section{Producción de inflorescencias}

Otra respuesta generalizada en los clones fue la producción inmediata de inflorescencias, asociada al brusco cambio de densidad provocado por el trasplante. En los primeros 45 días el $100 \%$ de los ramets produjeron flores, formándose hasta 4 inflorescencias sucesivas, a pesar de que en la población original de Hidalgo la floración no es un proceso frecuente. El número de flores por inflorescencia varía de 6 a 11, sobrepasando las inflorescencias la altura de las plantas. La frecuencia de floración disminuye en las generaciones más jóvenes.

\section{DISCUSIÓN}

Numerosos estudios tanto en ambientes controlados como naturales, enfatizan el alto potencial de crecimiento de $E$. crassipes, considerada como una de las especies más productivas (Penfound y Earle, 1948; Wolverton y McDonald, 1979; Lallana, 1980, 1981; Debusk et al., 1981). De acuerdo con Bock (1969), el incremento de la población sigue una relación exponencial de forma $\mathrm{W}=\mathrm{W}_{0} \mathrm{X}^{\mathrm{t}}$, en donde el peso o el número de plantas alcanzado (W) durante un período de tiempo dado $(t)$, depende del peso inicial $\left(\mathrm{W}_{0}\right)$ y de un factor de incremento $\left(\mathrm{X}=\mathrm{e}^{\mathrm{r}}\right)$. Sin embargo, este comportamiento "ideal" se presenta en condiciones de desarrollo sin restricciones, como ocurre en las primeras etapas del crecimiento de la población, ya que más tarde el incremento exponencial disminuye hasta alcanzarse una fase de estabilidad relativa asociada a factores tanto intrínsecos, debido al aumento de densidad de la propia población, así como a diversos factores extrínsecos, de tal manera que el crecimiento de la población no solo dependerá de la tasa intrínseca de incremento, sino también de un nuevo factor que marca el límite máximo de crecimiento (Harper, 1977).

El acelerado crecimiento de las poblaciones en un medio eutroficado, cuando $E$. crassipes es la especie pionera, como ocurre en el sitio Hidalgo durante la época cálida, marca un fuerte contraste al comparar el crecimiento de las poblaciones cuando se desarrollan en un medio cálido como el sitio Veracruz, en donde a pesar de que factores tales como la concentración de elementos nutritivos, temperatura, salinidad o $\mathrm{pH}$ no son adversos, la producción de unidades de crecimiento clonal se encuentra fuertemente limitada, siendo muy probable que la alta diversidad específica presente en la comunidad pueda desempeñar un papel determinante en el desarrollo de los clones.

Bajo condiciones de restricción para la producción de unidades de crecimiento clonal, los ramets modifican su patrón de crecimiento al mantener un arreglo mayor en el número de hojas funcionales. La inmediata producción de inflorescencias provocada 
BOLETÍN DE LA SOCIEDAD BOTÁNICA DE MÉXICO No. 45, 1983

TABLA 4. Parámetros, ajuste y significancia de la función logística que uescribe la producción de hojas para cada generación de ramets

Sitio

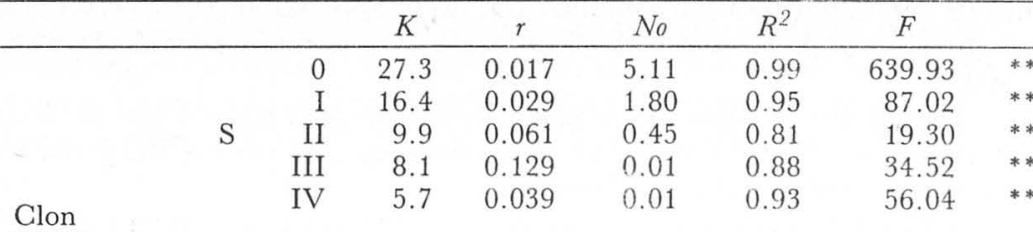

Hidalgo

Sitio

Hidalgo

Clon

\begin{tabular}{|c|c|c|c|c|c|}
\hline \multirow{5}{*}{ U } & 0 & 28.4 & 0.016 & 4.86 & 0.99 \\
\hline & I & 22.1 & 0.017 & 1.74 & 0.98 \\
\hline & II & 19.1 & 0.017 & 1.04 & 0.98 \\
\hline & III & 13.0 & 0.037 & 0.02 & 0.92 \\
\hline & IV & 13.0 & 0.039 & 0.03 & 0.98 \\
\hline
\end{tabular}

450.11

210.51

228.91

55.05

234.27

$\begin{array}{rrrrrrrr} & 0 & 24.4 & 0.022 & 7.50 & 0.95 & 62.39 & * * \\ \mathrm{~S} & \text { I } & 10.3 & 0.041 & 0.22 & 0.93 & 49.37 & * * \\ & \text { II } & 11.0 & 0.050 & 0.01 & 0.99 & 2037.49 & * * \\ & \text { III } & 10.8 & 0.028 & 0.01 & 0.94 & 56.29 & * *\end{array}$

Veracruz

$\begin{array}{rrrrrrrr} & 0 & 27.4 & 0.008 & 5.56 & 0.90 & 30.49 & * * \\ \mathrm{U} & \text { I } & 14.6 & 0.012 & 1.72 & 0.89 & 28.92 & * * \\ & \text { II } & 8.1 & 0.052 & 0.01 & 0.99 & 342.14 & * * \\ & \text { III } & 5.3 & 0.050 & 0.01 & 0.89 & 30.49 & * *\end{array}$

\begin{tabular}{|c|c|c|c|c|c|}
\hline 0 & 20.9 & 0.017 & 4.58 & 0.97 & 58.78 \\
\hline I & 19.3 & 0.027 & 1.18 & 0.97 & 39.70 \\
\hline II & 25.5 & 0.017 & 2.11 & 0.99 & 25.27 \\
\hline
\end{tabular}

Clon

Hidalgo

Veracruz

$\begin{array}{rrrrrrrr} & 0 & 9.2 & 0.031 & 3.80 & 0.93 & 28.71 & * * \\ \mathrm{U} & \text { I } & 24.0 & 0.018 & 1.69 & 0.98 & 97.63 & * * \\ & \text { II } & 23.2 & 0.018 & 1.37 & 0.99 & 123.46 & * * \\ & & & & & & & \\ & 0 & 25.3 & 0.011 & 9.74 & 0.95 & 39.82 & * * \\ \mathrm{~S} & \text { I } & 18.0 & 0.021 & 1.79 & 0.98 & 122.15 & * * \\ & \text { II } & 22.8 & 0.042 & 0.09 & 0.99 & 343.49 & * * \\ & & & & & & & \\ & 0 & 31.3 & 0.018 & 2.60 & 0.99 & 561.41 & * * \\ \mathrm{U} & \text { I } & 22.5 & 0.018 & 1.75 & 0.99 & 255.59 & * * \\ & \text { II } & 11.5 & 0.052 & 0.02 & 0.78 & 6.89 & * * \\ \end{array}$

Clon

Veracruz

por el transplante, reflejo de la gran plasticidad adaptativa de la especie, impide el entendimiento del comportamiento de las poblacionales en condiciones normales, no obstante, el mantenimiento de una unidad física entre los ramets parece ser un factor determinante para la supervivencia de las plantas en estado juvenil.

\section{AGRADECIMIENTOS}

Al doctor José Sarukhán por su constante estímulo y la valiosa sugerencia de la colaboración de la doctora Maxine Watson durante la fase de maduración de la idea. 


\section{DEMOGRAFÍA Y GRECIMIENTO DEL LIRIO ACUÁTICO}

De manera especial al M. en C. Exequiel Ezcurra y al doctor Rodolfo Dirzo por su entusiasta motivación, apoyo y revisión crítica del manuscrito. Al ingeniero José Sandoval y a la señorita Flora Barrios por las facilidades para realizar el trabajo de campo, así como al grupo de Vegetación Acuática por su participación.

El trabajo fue llevado a cabo con el subsidio económico del proyecto PCECNAL 790236 al Programa Nacional Indicativo de Ecología del Conacyt y gracias a la beca otorgada al primer autor a través del convenio UNAM-Conacyt.

\section{BIBLIOGRAFÍA}

ALMAZÁN, G. and C.E. BOYD. 1978. Effects of nitrogen levels on rates of oxigen consumption during decay of aquatic plants. Aquat. Bot. 5(2):119-126.

BATES, R.P. and J.F. HENTGES. 1976. Aquatic weeds - eradicate or cultivate? Econ. Bot. 30:39-50.

BAGNAL, L.O., R.L. SHIRLEY and J.F. HENTGES. 1973. Processing and Chemical Composition and Nutritive Value of Aquatic Weeds. Publicación 25, Florida Water Resourses, Research Center.

BAYO, V., V.H. LALLANA, E. LORENZATTI y M.C. MARTA. 1981. Evaluación cuantitativa de la vegetación acuática en islas del valle aluvial del río Paraná medio. Parte 1. Ecología 6:67-72.

BAZZAZ, F.A. and J.L. HARPER. 1977. Demographic analysis of the growth of Linum usitatissimum. New Phytol. 78:193-208.

BISHAI, H.M. 1960. Effect of Eichhornia crassipes on the hydrobiology of the Nile Water. Annual Repnrt Hydrobiological Research Unit, Univ. of Khartoum.

BOCK, J.H. 1969. Productivity of the water hyacinth Eichhornia crassipes (Mart) Solms. Ecology 50(3):460-464.

BOYD, C.E. 1968. Fresh water plants: A potential source of portein. Econ. Bot. 22:359-368.

1969. The nutritive value of three species of water weeds. Econ. Bot. 23:123-127.

1970. Vascular aquatic plants for mineral nutrient removal from polluted waters. Econ. Bot. 24:95-103.

1973. A bibliography of interest in the utilization of vascular aquatic plants. Econ. Bot. 26:74-84.

1976. Accumulation of dry matter, nitrogen and phosphorus by cultivated water hyacinths. Econ. Bot. 30:51-56.

CENTER, T.D. 1981. Samoedes albiguttalis a new biological agent for water hyacint. Aquatics 3(4):8-12.

COOLEY, T.N., M.H. GONZÁLEZ and D.F. MARTIN. 1978. Radio-magnese, -iron, and phosphorus uptake by water hyacinth and economic implications. Econ. Bot. 32(4):371-378.

COOLEY, T.N. and D.F. MARTIN. 1980. Diurnal variation of selected parametrs under waterhyacinths and in open water. Florida Sci. 43(1):26-32.

DEBUSK, T.A., M.D. HANISAK, L.D. WILLIAMS and J.H. RYTHER. 1981. Effects of seasonality and plants density on the productivity of some fresh-water macrophytes. Aquat. Bot. 10:133-142.

DUNIGAN, E.P., R.A. PHELAN and Z.H. SHAMSUDDIN. 1975. The use of water 
hyacinth to remove nitrogen and phosphorus from eutrophic water. Hyacinth Cont. J. 13:59-61.

FORMO, I.W. and A.D. WRIGHT. 1981. The biology of Australian weeds. 5. Eichhornia crassipes (Mart) Solms. J. Aus. Inst. Agric. Sci. 47:21-28.

GARCÍA, E. 1973. Modificación al sistema de clasificación climática de Köeppen. Inst. de Geografía, UNAM.

GAY, P.A. 1958. Eichhornia crassipes in the Nile of the Sudan. Nature 182 (4634):538-539.

1960. Ecological studies of E. crassipes Solms. in the Sudan I. Anaylisis of spread in the Nile. J. Ecol. 48:185-191.

GOPAL, B. and K.P. SHARMA. 1979. Aquatic weed control versus utilization. Econ. Bot. 33:340-346.

GUILlarmoD, A.J. 1979. Water Weeds in South Africa. Aquat. Bot. 6:377-391.

HALL, T.F. 1961. Principles of aquatic plant control. Adv. Pest. Control Res. $4: 211-247$.

HARPER, J.L. 1967. The regulation of numbers and mass in plant populations. In:R.C. Lewontin (ed.) Population Biology and Evolution. Syracuse Univ. Press. 1977. Population Biology of Plants. Academic Press. London. 1978. The demography of plants with clonal rowth. Verh. Kon. Nederland. Akad. Weten., Afd. Nat. 70:27-48.

and A.D. BELL. 1979. The population dynamics of growth form in organisms with modular construction. In: R.M. Anderson, B.D. Turner, L.R. Taylor (eds.) Population dynamics, 20th Symp. Brit. Ecol. Soc. Oxford. Blackwell Sci. Publ. HAYASHI, I., J.V. PANCHO and S. SASTROUTOMO. 1978. Preliminary reports on the buried seeds of floating islands and bottom of lake Rawa Pening, Central Java. Jap. J. Ecol. 28(4):325-333.

HITCHCOCK, A.E., P.W. ZIMMERMAN, H. KIRKPATRICK and T.T. EARLE. 1950. Growth and reproduction of water hyacinth and alligator weed and their control by means of ${ }_{2,4} \mathrm{D}$. Contr. Boyce Thompson Inst. 16:91-130.

JAIN, S.C. 1975. Aquatic weeds and their management in India. Hyacinth Contr. J. 13:6-8.

LALLANA, V.H. 1980. Productividad de Eichhornia crassipes (Mart) Solms. en una laguna isleña de la cuenca del río Paraná medio. II. Biomasa y dinámica de población. Ecología 5:1-16.

1981. Productividad de Eichhornia crassipes (Pontederiaceae) en una laguna isleña de la cuenca del río Paraná medio. I. Análisis de crecimiento. Bol. Soc. Argen. Bot. 20(1-2):99-107.

LITTLE, E.C.S. 1968. The control of water weeds. Weed Res. 8:79-105.

1968a. Handbook of utilization of aquatic plants. Food and Agriculture Organization of the United Nations. FAO, Rome.

LOT, A., A. NOVELO y A. QUIROZ. 1979. The Chinampa: an agricultural system that utilizes aquatic plants. J. Aquat. Plant Manage. 17:74-75.

McDONALD, R.C. and B.C. WOLVERTON. 1980. Comparative study of waste water lagoon with and without water hyacinth. Econ. Bot. 34:101-110.

ORNES, W.H. and D.L. SUTTON. 1975. Removal of phosphorus from static sawage eftluent by waterhyacinth. Hyacinth Cont. J. 13:56-58.

PENFOUND, W.T. and T.T. EARLE. 1948. The biology of water hyacinth. Ecol. Monogr. 18(4):447-472. 
PERKINS, B.D. 1974. Arthropods that stress water hyacinth. PANS 20(3):304-314. QUIROZ, F.A., M.G. MIRANDA y A. LOT. 1982. Uso potencial de algunas hidrófitas como abono verde en la zona chinampera de Xochimilco. Biótica 7(4):631-633. RAI, D.N. and JAYASHREE-DATTA-MUNSHI. 1979. The influence of thick floating vegetation ( $E$. crassipes) on the phisicochemical environment of a fresh water wetland. Hydrobiologia 62(1):65-70.

ROGER, H.H. and D.E. DAVIS. 1972. Nutrient removal by waterhyacinth. Weed Sci. 20(5):423-428.

ROOM, P.M., K.L.S. HARLEY, M.H. JULIEN, A.D. WRIGHT and I.W. FORMO. 1979. Progress towards biological control of aquatic weeds in Australia. Proceedings 7th. Asian-Pacific Weed Sci. Soc. Conf.

SANDERS, D.R., R.F. THERIOT and E.A. THERIOT. 1982. Organisms impacting waterhyacinth in Panama Canal. J. Aquat. Plant Manage. 20:22-29.

SINHA, S.N. and L.P. SINHA. 1969. Studies in use water hyacinth culture in oxidation ponds treating digested sugar wastes and effluent of septic tank. Envir. Healt 11:197-207.

SOERJANI, M., J.V. PANCHO and NGUYEN VAN-VUONG. 1975. Aquatic weed problems and control in southeast Asia. Hyacinth Cont. J. 13:2-3.

STAWINSKI, T.M. y O.H. MONROY. 1982. Metales tóxicos en el lirio acuático. En: II Congreso sobre Problemas Ambientales de México. COPEA-ENCB, México, D.F.

TIMMER, C.E. and L.W. WELDON. 1967. Evapotranspiration and pollution of water by water hyacinth. Hyacinth Cont. J. 6:34-37.

TRIVEDY, R.K., K.P. SHARMA, P.K. GOEL and B. GOPAL. 1978. Some ecological observations on floating islands. Hydrobiologia 60(2):187-190.

VIETMEYER, N.D. 1975. The beautiful blue devil. An IPPC Papers Reprinted from: The International Plant Protection Center, Oregon.

WHITE, J. 1979. The plant as a metapopulation. Ann. Rev. Ecol. Syst. 10:109-145.

WOLVERTON, B.C. and R.C. McDONALD. 1978. Nutritional composition of water hyacinth grown on domestic sewage. Econ. Bot. 32:363-370.

WOLVERTON, B.C. and R.C. McDONALD. 1979. Water hyacinth productivity and harvesting studies. Econ. Bot. 33:1-10.

and M.M. McKOWN. 1976. Waterhyacinth for removal phenols from polluted waters. Aquat. Bot. 2(3):191-202.

WOOTEN, J.W. and J.D. DODD. 1976. Growth of water hyacinths in treated sewage effluent. Econ. Bot. 30:29-37.

WRIGHT, H.D. 1980. Biological control of water hyacinth in Australia. In: Proc. V. Int. Symp. Biol. Contr. Weeds, Brisbane, Australia. 